\title{
Modulation of the longitudinal pumping in quantum spin systems
}

\author{
A. A. Zvyagin \\ Max-Planck-Institut für Physik komplexer Systeme, Noethnitzer Str., 38, D-01187 Dresden, Germany; \\ B.I. Verkin Institute for Low Temperature Physics and Engineering of the National Academy of Sciences of Ukraine, \\ Nauky Ave., 47, Kharkiv 61103, Ukraine; \\ and V.N. Karazin Kharkiv National University, Svobody Sqr. 4, Kharkiv 61022, Ukraine
}

(Received 27 September 2019; revised manuscript received 18 February 2020; accepted 1 April 2020; published 5 May 2020)

\begin{abstract}
The effect of the modulation of a high-frequency ac magnetic field parallel to the dc one (longitudinal pumping) by the lower-frequency ac field acting on quantum spin systems is considered for closed and open systems. For most magnetically ordered spin systems, the modulation causes the oscillation of the threshold of the parametric instability of spin waves as a function of the amplitude and the frequency of the modulation. On the other hand, our results for quantum spin systems with fermionic eigenstates imply that the modulating field causes the combined resonance and the modulation of the magnitude of time-dependent oscillations of the magnetization and the absorbed by the system power of the ac field for the closed system and for the open system in the dynamical and the steady-state regimes.
\end{abstract}

DOI: 10.1103/PhysRevB.101.174408

\section{INTRODUCTION}

During recent years the interest in the dynamics of quantum systems has grown considerably. Quantum many-body systems out of equilibrium usually do not follow the general principles of equilibrium systems. Standard ensembles of statistical mechanics use, as a rule, only few conserved values of the dynamical system and basically describe the behavior after relaxation. Instead, the time evolution of quantum-mechanical expectation values depends on time through a large number of parameters of the considered quantum many-body system. Studies of nonequilibrium dynamics of quantum many-body models are necessary for the fundamental understanding of how statistical physics emerges under the unitary time evolution. Such studies can inform us about the rates of spreading of correlations and entanglement in quantum many-body systems. Fundamental problems related to such time evolution include (but are not limited to), quantum quenches, equilibration, thermalization [1], prethermalization [2] in closed systems, generalized Gibbs ensemble [3], periodically driven quantum systems, dynamical quantum phase transitions [4], and the effect of damping on the quantum coherence in open systems.

Periodic changes of some parameters of a quantum system lead to a unitary time evolution, and the final (long time) state strongly depends on the type of system. Probably, the prime example of such a time evolution under the periodic driving

Published by the American Physical Society under the terms of the Creative Commons Attribution 4.0 International license. Further distribution of this work must maintain attribution to the author(s) and the published article's title, journal citation, and DOI. Open access publication funded by the Max Planck Society. is the use of the Floquet theorem [5] for many-body quantum systems [6]. Notice that, for studies of quantum condensedmatter many-body systems, powerful experimental techniques have been developed recently, including the ultrafast $\mathrm{THz}$ pulse technique [7], and experiments in pulsed fields [8]. On the other hand, studies of the time evolution of manybody systems out of equilibrium are also very important in the context of experiments on ultracold atoms, trapped ions, or superconducting qubits in quantum computers [9]. Such synthetic quantum many-body systems can serve as a very good testing ground for the exploration of nonequilibrium dynamics, because the coherence there is maintained for much longer times than for usual condensed matter.

Unlike higher-dimensional models, their low-dimensional quantum spin counterparts permit us to obtain exact (nonperturbative) results [10]. The necessity of nonperturbative studies in one-dimensional quantum systems is caused by the enhanced quantum and thermal fluctuations there, due to the features in the density of states [11]. It is known that the one-dimensional quantum spin models can describe not only real one-dimensional spin systems [12], but also topological superconductors $[13,14]$ and ultracold atoms in one-dimensional optical traps [15]. Low-dimensional quantum spin models often reveal properties of quantum spin liquids. In the latter the magnetic order is suppressed down to the lowest temperatures due to the frustration of spinspin interactions and/or enhanced quantum fluctuations in low-dimensional systems [16]. In many quantum spin liquids emergent magnetic excitations are fermions (as a rule they carry fractionalized spin), instead of magnons (bosons, which carry spin 1) for ordered magnetic systems. Notice that while bosons have their classical counterparts, fermions, instead, have a totally quantum nature.

Modulation of the periodic driving in quantum many-body systems can provide additional very important information 
about the nature of the time evolution of such a system out of equilibrium for both closed systems, where only dynamical processes emerge, and in open systems, where the damping is caused by the processes, which take the energy from the considered system. In this paper we study the effect of the modulation of a high-frequency ac magnetic field parallel to the dc one (the so-called longitudinal pumping) by the lowerfrequency ac field, acting on quantum systems. We show that the modulating field produces the combined resonance at the frequency of the pumping and (several) frequencies of the modulating field, together with the modulation of characteristics of the considered systems as a function of the magnitude and the frequency of the modulating field. For most of magnetically ordered spin systems, the modulation of the longitudinal pumping yields the oscillation of the threshold of the parametric instability of spin waves as a function of the amplitude and the frequency of the modulation. For quantum spin systems with fermionic eigenstates, which carry fractional spin (characteristic for the wide class of quantum spin liquids), the modulating field causes the modulation of the time-dependent oscillations of the magnetization and the absorbtion by the system power of the ac field, unlike the exponential growth of the number of spin waves for magnetically ordered systems. Two main examples of the considered quantum spin systems are spin-1/2 chains and the honeycomb Kitaev spin model (in the ground state). We have shown that the magnetic moment for the closed system oscillates in time with the combined frequency of the pumping and $n$ frequencies of the modulation, and the smaller Rabi-like frequency. Similar behavior persists in the dynamical regime of the open system. On the other hand, in the steady-state regime of the open system, the Rabi-like oscillations are damped. Magnitudes of all those time-dependent oscillations are modulated by the modulation ac field. The power of the ac field, absorbed by the quantum system, also oscillates as a function of the magnitude and the frequency of the modulating field.

\section{MODULATION OF THE LONGITUDINAL PUMPING BY THE LOWER-FREQUENCY FIELD}

Let us start the consideration of the problem with the Hamiltonian of the quantum spin system,

$$
\mathcal{H}_{0}=-\sum_{i j}\left(J_{x} S_{i}^{x} S_{j}^{x}+J_{y} S_{i}^{y} S_{j}^{y}+J_{z} S_{i}^{z} S_{j}^{z}\right)-g \mu_{B} H \sum_{j} S_{j}^{z},
$$

where $J_{x}, J_{y}$, and $J_{z}$ are exchange integrals, and $S_{j}^{x}, S_{j}^{y}$, and $S_{j}^{z}$ are operators of $x, y, z$ projectors of the quantum spins situated at the sites $j, g$ is the $g$ factor, $\mu_{B}$ is the Bohr magneton, and $H$ is the external dc magnetic field. Formally we can use any lattice and any values of $i$ and $j$; however, in reality the exchange interaction is often limited by the nearest-neighbor coupling. Let us act on the considered spin system by the ac magnetic field polarized linearly, with the polarization parallel to the direction of the dc field (so-called longitudinal pumping). Suppose also, that the high-frequency ac magnetic field is modulated by the low-frequency field. The Hamiltonian of the considered system becomes $\mathcal{H}=\mathcal{H}_{0}+\mathcal{H}_{t}$, where

$$
\begin{aligned}
\mathcal{H}_{t} & =-g \mu_{B} h(t) \sum_{j} S_{j}^{z} \\
& \equiv-g \mu_{B}\left[h \cos (\omega t)+h_{m} \cos (\Omega t)\right] \sum_{j} S_{j}^{z},
\end{aligned}
$$

where $\omega$ and $h$ are the frequency and the magnitude of the longitudinal pumping with $\hbar \omega \sim J_{x, y, z}, g \mu_{B} H$. In the standard situation in experiments, the magnitude of the pumping is much smaller than the frequency, $g \mu_{B} h \ll \hbar \omega$. $\Omega$ and $h_{m}$ are the frequency and the magnitude of the modulating rf magnetic field. For standard spin systems the resonance frequency of the ac field is usually from several $\mathrm{GHz}$ to several $\mathrm{THz}$, i.e., it is in the radio frequency range, or in the infrared or far-infrared range. The frequency of the modulating field in experiments is much smaller than that of the longitudinal pumping $\Omega \ll \omega$, i.e., it is the rf field. In our consideration we do not use such a strong inequality for the value of the modulation frequency, considering only $\Omega<\omega$.

\section{A. Dynamics and kinetics of quantum closed and open systems}

To describe the dynamics of our quantum system we can find the solution of the nonstationary Schrödinger equation for the wave function for the pure system (or the Liouville equation for the density matrix for the mixed system), and then average the considered operator with the obtained timedependent wave function or density matrix. The Liouville equation for density matrix $\rho$ has the form

$$
i \hbar \dot{\rho}=[\mathcal{H}, \rho],
$$

where [.,.] denotes the commutator. In the pure state the Schrödinger equation for the pure state wave function is used. Such a behavior is characteristic for a closed system (for the pure system).

As a rule, however, the spin system is not isolated. There exist processes, which take the energy from the system, i.e., relaxation processes. The reason for the relaxation of the density matrix is the interaction of the considered system with some environment; such an interaction takes energy from the system. In that case the considered system is the open one. For the studied quantum spin system the lattice (i.e., the elastic subsystem of the crystal) can serve as such an environment. The dynamics of the density matrix of the open system for general Markovian processes is described by the Lindblad master equation [17] (here we write it in the diagonal form)

$$
i \hbar \dot{\rho}=[\mathcal{H}, \rho]+i \sum_{j=1}^{N^{2}-1} \gamma_{j}\left(\mathcal{L}_{j} \rho \mathcal{L}_{j}^{\dagger}+\frac{1}{2}\left\{\mathcal{L}_{j}^{\dagger} \mathcal{L}_{j}, \rho\right\}\right),
$$

where $N$ is the dimension of the system, $\{.,$.$\} denotes the$ anticommutator, and the orthonormal and traceless operators $\mathcal{L}_{j}$ are the Lindblad (jump) operators. For $\gamma_{j}=0$ the Lindblad equation is, obviously, the Liouville equation. In the model of random collisions [18], one can write the Lindblad operators as $\mathcal{L}_{j}=\sqrt{\left(\rho_{0}\right)_{j j}}|j\rangle\left\langle j^{\prime}\right|$, and suppose that all $\gamma_{j}$ are equal, which yields

$$
i \hbar \dot{\rho}=[\mathcal{H}, \rho]+i \hbar \gamma\left(\rho_{0}-\rho\right) .
$$


Such a form of the master equation was first suggested by Karplus and Schwinger [19]. It was used to describe the relaxation processes of quantum systems under the action of the ac electromagnetic field. It describes the interaction of the considered system with the bath, with the relaxation of the density matrix to $\rho_{0}$ in the steady state.

The approximation used implies equal relaxation times for all eigenmodes of the system. It is equivalent to the Bloch form of relaxation in the theory of the nuclear magnetic resonance [20]. Two relaxation times as in the Bloch approach can be easily introduced in the above scheme by using different relaxation rates for diagonal and nondiagonal components of the density matrix. One can also generalize the approach using, e.g., Torrey's phenomenological theory [21], which adds diffusion processes to the Bloch equations. It is possible to show that the effect of the linear relaxation in the Bloch form is similar to the effect of the relaxation in the Landau-Lifshitz form for magnetic systems [22]. Here we are interested mostly in the homogeneous response and can neglect the spatial dependence of relaxation. The substitution $\rho^{\prime}=\rho \exp (\gamma t)$ yields

$$
i \hbar \dot{\rho}^{\prime}=\left[\mathcal{H}, \rho^{\prime}\right]+i \hbar \gamma \rho_{0} \exp (\gamma t) .
$$

Naturally, it is possible to use other forms of the Lindblad operators. However, the choice of those jump operators is not very essential for the goal of the present study: the effect of the modulation of the parametric pumping. Different forms of those operators can, in principle, yield results different from the obtained (see below) results. However, we believe that the Bloch-Karplus-Schwinger-like form of the relaxation is generic for spin systems under periodic time-dependent perturbations, and our results describe the generic situation with the modulation of the parametric pumping in the considered systems.

\section{B. Effective Hamiltonian}

Then let us transfer the explicit time dependence of the Hamiltonian to the exchange-caused terms by the unitary transformation $\rho^{\prime}=U_{1} \rho_{1} U_{1}^{-1}$ with

$$
\begin{aligned}
U_{1}= & \exp \left(i \frac{g \mu_{B}}{\hbar} \int h(t) d t \sum_{j} S_{j}^{z}\right), \\
i \hbar \dot{\rho}_{1}= & {\left[\mathcal{H}_{1}, \rho_{1}\right]+i \hbar \gamma U_{1}^{-1} \rho_{0} U_{1} \exp (\gamma t), } \\
\mathcal{H}_{1}= & -\sum_{i j}\left[\left(\frac{J_{x}+J_{y}}{2} S_{i}^{+} S_{j}^{-}\right.\right. \\
& +\frac{J_{x}-J_{y}}{2} \exp \left[i \frac{2 g \mu_{B}}{\hbar} \int h(t) d t\right] S_{i}^{+} S_{j}^{+} \\
& \left.+ \text {H.c. })+J_{z} S_{i}^{z} S_{j}^{z}\right]-g \mu_{B} H \sum_{j} s_{j}^{z},
\end{aligned}
$$

where $S_{j}^{ \pm}=S_{j}^{x} \pm i S_{j}^{y}$. Such a situation is known as the parametric pumping (the parameters of the Hamiltonian depend periodically on time).
Then we can use the standard series

$$
\exp [i z \sin (\omega t)]=\sum_{n=-\infty}^{\infty} J_{n}(z) \exp (i n \omega t),
$$

where $J_{n}(z)$ is the Bessel function. For $g \mu_{B} h \ll \hbar \omega$ we can approximate $J_{0}\left(2 g \mu_{B} h / \hbar \omega\right) \approx 1$ and $J_{1}\left(2 g \mu_{B} h / \hbar \omega\right) \approx$ $\left(2 g \mu_{B} h / \hbar \omega\right)$. Then the time dependence can be approximated as

$$
\begin{aligned}
\exp \left[i \frac{2 g \mu_{B}}{\hbar} \int h(t) d t\right] \approx & {\left[1+i \frac{2 g \mu_{B} h}{\hbar \omega} \sin (\omega t)\right] } \\
& \times \sum_{n=-\infty}^{\infty} J_{n}(z) \exp (i n \Omega t),
\end{aligned}
$$

where $z=2 g \mu_{B} h_{m} / \hbar \Omega$. Now taking into account that $g \mu_{B} h \ll \hbar \omega$ we keep in $\mathcal{H}_{1}$ only the terms which do not depend on $h$ and the linear in $g \mu_{B} h / \hbar \omega$ terms, see below. Notice that all above procedures can be applied to the spin system of any space dimension and of any value of the site spin $S$. These results can be obviously generalized to spin-spin interactions of any radius.

\section{Quantum systems with bosonic excitations}

Now our aim is to diagonalize the part of $\mathcal{H}_{1}$ which does not depend on $h$. It is impossible in general to study the modulated parametric pumping for the general quantum spin system. The standard way to study its low-energy dynamics is the spin-wave approximation. We can use, e.g., the HolsteinPrimakoff representation of spin operators via bosonic ones [23]. The operators of spin projections can be approximately presented as

$$
S_{j}^{z}=S-b_{j}^{\dagger} b_{j}, \quad S_{j}^{+} \approx \sqrt{2 S} b_{j}, \quad S_{j}^{-} \approx \sqrt{2 S} b_{j}^{\dagger},
$$

with $b_{j}$ and $b_{j}^{\dagger}$ being bosonic operators of destruction and creation. After the Fourier and Bogolyubov transformations, the Hamiltonian $\mathcal{H}_{1}$ (up to a constant term) takes the form

$$
\begin{aligned}
\mathcal{H}_{1} \approx & \sum_{\mathbf{k}}\left(\epsilon_{\mathbf{k}} c_{\mathbf{k}}^{\dagger} c_{\mathbf{k}}+\frac{g \mu_{B} h}{\hbar \omega} \sum_{n=-\infty}^{\infty} J_{n}(z)\right. \\
& \left.\times\left[\mathcal{B}_{\mathbf{k}} e^{[i(\omega+n \Omega) t]} c_{\mathbf{k}} c_{-\mathbf{k}}+\text { H.c. }\right]\right),
\end{aligned}
$$

where $\epsilon_{\mathbf{k}}=\left\{\left[g \mu_{B} H+2 S J_{z}-S\left(J_{x}+J_{y}\right) \cos (\mathbf{k})\right]^{2}-\right.$ $\left.\left|\mathcal{B}_{\mathbf{k}} J_{0}(z)\right|^{2}\right\}^{1 / 2}$ is the energy of a magnon, $c_{\mathbf{k}}$ and $c_{\mathbf{k}}^{\dagger}$ destroys or creates the magnon, and $\mathcal{B}_{\mathbf{k}}=-i S\left(J_{x}-J_{y}\right) \sin \mathbf{k}$ [here the intersite distances are supposed to be unity, and we use the shorthand notation $f(\mathbf{k})=f\left(k_{x}, k_{y}, k_{z}\right)$ ]. The condition of the resonance $2 \epsilon_{\mathbf{k}}=\hbar(\omega+n \Omega)$ is determined by the smallness of the magnitude $h, g \mu_{B} h \ll \hbar \omega$. We drop the terms like $\{\exp [-i \omega+n \Omega) t] c_{\mathbf{k}} c_{-\mathbf{k}}+$ H.c. $\}$, using so-called resonance approximation [24]. It means that we consider exactly terms, explicitly dependent on time, which produce the nonzero contribution to the linear response. The remaining terms with an explicit time dependence can be omitted due to the smallness of the magnitude of the ac magnetic field $h$ (their contribution can be, in principle, calculated in the framework of the perturbation theory). 
The same result, i.e., Eq. (11), can be obtained within the macroscopic approach for the magnetically ordered system by using the spin-wave approximation [25]. Namely, suppose that the considered spin system is magnetically ordered. For the magnetically ordered system we can approximately replace the operators of spin projections in Eq. (25) by their average values. It means that the quantum spins are replaced by the classical vectors of the site magnetic moments (due to the magnetic ordering), and the quantum Hamiltonian is replaced by the classical density of energy. Then, dynamics of the classical vectors of the magnetic moments (i.e., of the order parameters of the magnetically ordered system) is described by the Landau-Lifshitz equation of motion. There, magnetic moments move in the effective field, which is the variation of the density of the energy with respect to related magnetic moments. This approach is equivalent to the use of the mean-field approximation in quantum mechanics [26]. Suppose that, due to $g \mu_{B} H>S J_{x}, S J_{y}, S J_{z}$ the site magnetic moments of the considered ordered magnetic system are directed mostly along the $z$ axis. Then we can consider small deviations of magnetic moments (i.e., spin waves) and linearize the obtained equations of motion for those small deviations. The density of the energy, bilinear in such small deviations, can be diagonalized by the Fourier transform and the unitary transformation. Spin waves behave like bosons. They are often considered to be equivalent to magnons in magnetically ordered systems. For classical vectors of magnetic moments in magnetically ordered systems the number of states of their projections is infinite, unlike the finite number for a quantum spin. It is the reason why magnons in the magnetically ordered systems behave as bosons. Finally, using the resonance approximation, we get the density of the energy of spin waves in the same form as Eq. (11).

Now we use the unitary transformation $U_{2}=\exp \{[-i(\omega+$ $\left.n \Omega) t / 2] \sum_{\mathbf{k}} c_{\mathbf{k}}^{\dagger} c_{\mathbf{k}}\right\}$, with $\rho_{1}=U_{2} \rho_{2} U_{2}^{-1}$, which yields

$$
\begin{aligned}
i \hbar \dot{\rho}_{2}= & {\left[\mathcal{H}_{2}, \rho_{2}\right]+i \hbar \gamma U_{2}^{-1} U_{1}^{-1} \rho_{0} U_{1} U_{2} \exp (\gamma t), } \\
\mathcal{H}_{2}= & \sum_{\mathbf{k}}\left[\left(\epsilon_{\mathbf{k}}-\frac{\hbar(\omega+n \Omega)}{2}\right) c_{\mathbf{k}}^{\dagger} c_{\mathbf{k}}\right. \\
& \left.+\frac{g \mu_{B} h}{\hbar \omega} \sum_{n} J_{n}(z)\left(\mathcal{B}_{\mathbf{k}} c_{\mathbf{k}} c_{-\mathbf{k}}+\text { H.c. }\right)\right] .
\end{aligned}
$$

There can be several values of $n=0, \pm 1, \pm 2, \ldots$ for which the value $\hbar(\omega+n \Omega) / 2$ is inside the band of magnons $\epsilon_{k}$. $\mathcal{H}_{2}$ is the quadratic form of Bose operators. It follows from the equations of motion for $c_{\mathbf{k}}$ and $c_{-\mathbf{k}}^{\dagger}$ that the increment (decrement) of the time dependence of the $c_{\mathbf{k}}$ and $c_{-\mathbf{k}}^{\dagger}$ (and, hence, of the average with the density matrix or with the ground-state wave function at $T=0$ of the number of magnons $\left\langle c_{\mathbf{k}}^{\dagger} c_{\mathbf{k}}\right\rangle$ ) depends on whether the value $-\left\{\varepsilon_{\mathbf{k}}-[\hbar(\omega+n \Omega) / 2]\right\}^{2}+$ $\left|g \mu_{B} h \mathcal{B}_{\mathbf{k}} J_{n}(z) / \hbar \omega\right|^{2}$ is larger (smaller) than $(\hbar \gamma)^{2}$. In resonance we can neglect the term $\left\{\varepsilon_{\mathbf{k}}-[\hbar(\omega+n \Omega) / 2]\right\}^{2}$. Then for any $\gamma$ there exists the threshold value of the magnitude of the ac field $h_{c}$ : For the magnitude of the ac field larger than that threshold value, $h>h_{c}$, the number of magnons in the system grows with time exponentially (and the linear relaxation cannot limit such a growth). Such a parametric instability [25] is observed in many magnetic systems [27]. The threshold value of the field is [28]

$$
h_{c}=h_{c 0} \frac{\epsilon_{\mathbf{k}}}{\left.\epsilon_{\mathbf{k}}\right|_{z \rightarrow 0}}\left[\max _{n}\left|J_{n}(z)\right|\right]^{-1},
$$

where $h_{c 0}$ is the threshold value in the absence of the modulating field, at $h_{m}=0$. We see that the threshold value oscillates as a function of $h_{m} / \Omega$. The maximum values of $h_{c}$ as a function of the modulation frequency and/or magnitude are determined by $\left|J_{n}(z)\right|=\left|J_{n+1}(z)\right|$, and the condition for minimum values is $J_{n}(z)=0$. Such oscillations of the threshold of the parametric instability under the modulated longitudinal pumping were observed in magnetically ordered systems [29].

\section{Quantum spin systems with fermionic eigenstates}

From now on we limit our consideration by the lowdimensional quantum spin systems with site spins $S=1 / 2$, for which we can use the fermionic representation of spin operators, unlike the bosonic one in the Holstein-Primakoff or quasiclassical approximation used above. For example, for the spin- $1 / 2$ chain with nearest-neighbor interactions after the well-known Jordan-Wigner transformation [30], and the Fourier transformation, the time-independent part of the Hamiltonian $\mathcal{H}_{1}$ can be written as

$$
\begin{aligned}
\mathcal{H}_{\text {lind }}= & -\frac{N\left(J_{z}+2 g \mu_{B} H\right)}{4} \\
& +\sum_{k}\left[\left(g \mu_{B} H-\frac{J_{x}+J_{y}}{2} \cos (k)+J_{z}\right) d_{k}^{\dagger} d_{k}\right. \\
& \left.+i \frac{J_{x}-J_{y}}{4} \sin (k) J_{0}(z)\left(d_{-k} d_{k}-\text { H.c. }\right)\right] \\
& -\frac{J_{z}}{N} \sum_{k_{1}+k_{2}=k_{3}+k_{4}} \cos \left(k_{1}-k_{4}\right) d_{k_{1}}^{\dagger} d_{k_{2}}^{\dagger} d_{k_{3}} d_{k_{4}},
\end{aligned}
$$

where the fermion operators $d_{k}\left(d_{k}^{\dagger}\right)$ destroy (create) the spinless fermion with the quasimomentum $k$, and $N$ is the number of sites in the chain (the interspace distance is taken to be unity). Remember that we consider the magnitude of the pumping field to be much smaller than the exchange constants, $g \mu_{B} h \ll J_{x}, J_{y}, J_{z}$. Hence we can take into account the interaction between fermions in the dynamical mean-field-like approximation; see, e.g., Ref. [31]. Notice that for $J_{z}=0$ the fermion form of the Hamiltonian (14) is exact. It corresponds to the $X Y$ spin chain or, in our particular case, to the transverse field Ising chain.

In the main approximation with respect to the small parameter $g \mu_{B} h / \hbar \omega$ we will not take into account the pumping field $h$ in the self-consistency equations. Our answers, see below, imply that the results (the change of the magnetic moment due to the ac field and the absorbed power of the ac field) even in the main (zero) approximation in the self-consistency equations, are already proportional to that small parameter, or the square of it. Hence, the next order in $g \mu_{B} / \hbar \omega$ in the selfconsistency equations will produce only small in $g \mu_{B} h / \hbar \omega$ corrections to the obtained results.

Let us introduce the parameters

$$
\begin{aligned}
& s=\left\langle S_{j}^{z}\right\rangle, \quad r=2\left\langle S_{j}^{x} S_{j+1}^{x}+S_{j}^{y} S_{j+1}^{y}\right\rangle, \\
& q=2\left\langle S_{j}^{x} S_{j+1}^{x}-S_{j}^{y} S_{j+1}^{y}\right\rangle,
\end{aligned}
$$


which satisfy the self-consistency equations

$$
\begin{aligned}
& s=\frac{1}{2 N} \sum_{k} \frac{A_{k}}{\varepsilon_{k}} \tanh \left(\frac{\varepsilon_{k}}{2 k_{B} T}\right), \\
& r=-\frac{1}{N} \sum_{k} \frac{A_{k} \cos (k)}{\varepsilon_{k}} \tanh \left(\frac{\varepsilon_{k}}{2 k_{B} T}\right), \\
& q=-\frac{i}{N} \sum_{k} \frac{B_{k} \sin (k) J_{0}(z)}{\varepsilon_{k}} \tanh \left(\frac{\varepsilon_{k}}{2 k_{B} T}\right),
\end{aligned}
$$

where

$$
\begin{aligned}
& A_{k}=g \mu_{B} H+2 s J_{z}-\left(\frac{J_{x}+J_{y}}{2}-r J_{z}\right) \cos (k), \\
& B_{k}=-i\left(\frac{J_{x}-J_{y}}{2}+q J_{z}\right) \sin (k), \\
& \varepsilon_{k}=\sqrt{A_{k}^{2}+\left|B_{k}\right|^{2} J_{0}^{2}(z)} .
\end{aligned}
$$

In Eq. (17), $T$ is the temperature, $k_{B}$ is the Boltzmann constant, and brackets denote the Gibbs averaging with the Hamiltonian $\mathcal{H}_{\text {lind }} \approx \sum_{k} \varepsilon_{k} a_{k}^{\dagger} a_{k}+C$, where the fermion operators $a_{k}\left(a_{k}^{\dagger}\right)$ are destruction (creation) operators, in which $\mathcal{H}_{1 \text { ind }}$ is diagonal in the dynamical mean-field-like approximation, and $C$ is the operator-independent value. Then the Hamiltonian $\mathcal{H}_{1}$ can be written in the resonance approximation as

$$
\begin{aligned}
\mathcal{H}_{1} \approx & \sum_{k}\left[\varepsilon_{k} a_{k}^{\dagger} a_{k}+i \frac{g \mu_{B} h}{\hbar \omega} \sum_{n=-\infty}^{\infty} J_{n}(z)\right. \\
& \left.\times\left(B_{k} a_{-k} a_{k} \exp [i(\omega+n \Omega) t]-\text { H.c. }\right)\right] .
\end{aligned}
$$

Here $A_{k}, B_{k}$, and $\varepsilon_{k}$ are determined from Eqs. (17) via the solution of self-consistency equations (16). In the general case of nonzero $J_{x}, J_{y}, J_{z}$ we can solve Eqs. (16) analytically, e.g., for high temperatures $k_{B} T \gg \max \left(\varepsilon_{k}\right)$. The solution is

$$
\begin{aligned}
s= & \frac{g \mu_{B} H}{4 k_{B} T-2 J_{z}}, \\
r \approx & \frac{J_{x}+J_{y}}{4 k_{B} T+J_{z}}, \quad q \approx \frac{\left(J_{x}-J_{y}\right) J_{0}(z)}{4 k_{B} T-J_{z}}, \\
A_{k} \approx & g \mu_{B} H\left(1+\frac{J_{z}}{2 k_{B} T}\right) \\
& -\frac{\left(J_{x}+J_{y}\right) \cos (k)}{2}\left(1-\frac{J_{z}}{k_{B} T}\right), \\
B_{k} \approx & -i \frac{\left(J_{x}-J_{y}\right) \sin (k) J_{0}(z)}{2}\left(1+\frac{J_{z}}{4 k_{B} T}\right),
\end{aligned}
$$

where $J_{z} \ll k_{B} T$. However, more interesting is to study the low-temperature regime, in which correlations of the one-dimensional spin system can manifest themselves. Unfortunately, even in the ground state the solution of Eqs. (16) is complicated (it can be presented as the combination of elliptic integrals). However, the simple solution, as we will show below, reveals some main features of exact results for spin- $1 / 2$ chains, at least in the ground state. We can consider the case $H=0$ (with $s=0$ ), in which the following relation holds

$$
\frac{\left(J_{x}-J_{y}\right) J_{0}(z)}{2}+q J_{z}= \pm \frac{J_{x}+J_{y}}{2} \mp r J_{z} .
$$

These relations mean that $(r+q) J_{z}=J_{x}\left[1-J_{0}(z)\right] / 2+$ $J_{y}\left[1+J_{0}(z)\right] / 2$ for the plus sign, and $(r-q) J_{z}=J_{x}[1+$ $\left.J_{0}(z)\right] / 2+J_{y}\left[1-J_{0}(z)\right] / 2$ for the minus sign. The selfconsistency equations are simplified to

$$
r= \pm q=\frac{1}{2} \tanh \left(\frac{J_{x}+J_{y} \mp 2 r J_{z}}{4 k_{B} T}\right), \quad s=0,
$$

with $q=r$ for the plus sign, and $q=-r$ for the minus sign. The transcendental equations (21) can be solved graphically, and the solution exists for any temperature range. It is easy to show that the solution leads to the onset of a critical temperature, below which the magnetic ordering can take place. It is, of course, the artifact of the mean-field nature of the dynamical mean-field-like consideration. The critical nonzero temperature must not exist for a one-dimensional spin system with the nearest-neighbor interactions with gapless excitations [11]. Here, on the other hand, excitations are gapped. We know that the Ising chain and the $X Y$ chain reveal ordering in the ground state [32]. For $T=0$ the simple solution of the self-consistency equation (21) corresponds to $r=q=1 / 2$, or $r=-q=1 / 2$, related to $J_{z}=J_{x}\left[1-J_{0}(z)\right] / 2+J_{y}[1+$ $\left.J_{0}(z)\right] / 2$, or $J_{z}=J_{x}\left[1+J_{0}(z)\right] / 2+J_{y}\left[1-J_{0}(z)\right] / 2$. The solution manifests the spin ordering for $x(y)$, components of spins at $T=0$, expected [32] for $J_{y} \neq J_{x}$. Such an ordering is similar to those of the Ising or $X Y$ chains [32]. Notice, however, that mean field features of the used dynamical approximation imply mean-field values of correlation exponents. The renormalization of those exponents can be taken into account, e.g., in the bosonization approach [33]. The main features of the above shown results will be kept, though.

We can also consider the $S=1 / 2$ honeycomb Kitaev model [34] for the case $H=0$. We rewrite the timeindependent part of the Hamiltonian $\mathcal{H}_{1}$ exactly by using the transformation to fermion operators of creation and destruction $d^{\dagger}$ and $d$ (for our purpose it is convenient to use the Dirac representation for fermion operators). It is the two-dimensional generalization $[35,36]$ of the Jordan-Wigner transformation [30]. To represent spin operators with spinless fermion operators we can use [35]

$$
\begin{aligned}
S_{j, l}^{+} & =d_{j, l}^{\dagger} \exp \left[\sum_{i, k<j} d_{i, k}^{\dagger} d_{i, k}+\sum_{i<l} d_{i, l}^{\dagger} d_{i, l}\right], \\
S_{j, l}^{z} & =d_{j, l}^{\dagger} d_{j, l}-1 / 2, \\
S_{j, l}^{-} & =\left(S_{j, l}^{+}\right)^{\dagger} .
\end{aligned}
$$

Here the indexes $j, l$ denote the column and row of the brick lattice equivalent to the honeycomb lattice [35]. For each pair of Dirac fermion operators $d$ and $d^{\dagger}$ two Majorana fermion operators $a$ and $f$ can be defined as $a_{j, l}=i\left(d_{j, l}^{\dagger}-d_{j, l}^{\dagger}\right)$ and $f_{j, l}=d_{j, l}^{\dagger}+d_{j, l}$ when $j+l$ is even, and $a_{j, l}=d_{j, l}^{\dagger}+d_{j, l}$ and $f_{j, l}=i\left(d_{j, l}^{\dagger}-d_{j, l}\right)$ when $j+l$ is odd. It turns out that the values $\alpha_{j}=i f_{j, l} f_{j, l+1}$ defined on each vertical bond are conserved [34,35]. The Hamiltonian $\mathcal{H}_{0}$ can be written (using more convenient enumeration of bonds instead of columns 
and rows) as the Hamiltonian of the Fermi gas on the brick lattice with the site-dependent chemical potential

$$
\begin{aligned}
\mathcal{H}_{0}= & \sum_{j}\left[J_{x}\left(d_{j}^{\dagger}+d_{j}\right)\left(d_{j+\hat{x}}^{\dagger}-d_{j+\hat{x}}\right)+J_{y}\right. \\
& \left.\times\left(d_{j}^{\dagger}+d_{j}\right)\left(d_{j+\hat{y}}^{\dagger}-d_{j+\hat{y}}\right)+2 J_{z} \alpha_{j}\left(2 d_{j}^{\dagger} d_{j}-1\right)\right],
\end{aligned}
$$

where $j$ stands for the position of the $z$ bond, $\hat{y}$ connects two $z$ bonds, and crosses a $y$ bond (similar definition holds for $\hat{x}), \alpha_{j}= \pm 1\left(\alpha_{j}\right.$ commutes with $d_{j^{\prime}}$ and $d_{j^{\prime}}^{\dagger}$ for any $j$ and $\left.j^{\prime}\right)$. Notice that similar result (with different notations) was obtained in the original paper [34], i.e., the result does not depend on the numeration, as must be. This transformation is exact, and it is valid for any $J_{x}, J_{y}, J_{z}$. In the sectors with fixed $\alpha_{j}$ the diagonal form of the Kitaev model can be obtained after the Fourier and Bogolyubov transformations. Performing similar calculations for the time-independent part of $\mathcal{H}_{1}$, we see that it has the BCS-like form with the energy

$$
\varepsilon_{\mathbf{k}}=\sqrt{A_{\mathbf{k}}^{2}+\left|B_{\mathbf{k}}\right|^{2} J_{0}^{2}(z)}
$$

where $A_{\mathbf{k}}= \pm J_{z}+J_{x} \cos k_{x}+J_{y} \cos k_{y}$ and $B_{\mathbf{k}}=i\left[J_{x} \sin k_{x}+\right.$ $\left.J_{y} \sin k_{y}\right]$. The spectrum is gapless for $\left|J_{x}-J_{y}\right| \leqslant J_{z} \leqslant J_{x}+$ $J_{y}$, and gapped otherwise.

We see that, formally (up to the definition of the wave vector; from now on we denote $\mathbf{k} \rightarrow k$ for simplicity), the Hamiltonian $\mathcal{H}_{0}$ is written in the same form for the quantum spin chain and for the Kitaev honeycomb spin model (notice the $Z_{2}$ field $\alpha_{j}$ present in the latter). Then the Hamiltonian $\mathcal{H}_{1}$ can be written in terms of normal modes of the Hamiltonian $\mathcal{H}_{\text {1ind }}$, cf. [37],

$$
\begin{aligned}
\mathcal{H}_{1} \approx & \sum_{k}\left(\varepsilon_{k} a_{k}^{\dagger} a_{k}+i \frac{g \mu_{B} h}{\hbar \omega} \sum_{n} J_{n}(z)\right. \\
& \left.\times\left\{B_{k} a_{-k} a_{k} \exp [i(\omega+n \Omega) t]-\text { H.c. }\right\}\right) .
\end{aligned}
$$

Notice that the pumping term related to the operator $\sum_{j} S_{j}^{z}$ can change homogeneously the sign of $\alpha_{j}$ of the original Hamiltonian [37].

Here we have also used the resonance approximation, i.e., we have considered exactly only terms explicitly dependent on time and which produce the nonzero contribution to the linear response (25). When doing so we fixed the value of $n$ by the resonance condition $2 \varepsilon_{k}=\hbar(\omega+n \Omega)$. We have dropped the terms like $a_{-\mathbf{k}} a_{\mathbf{k}} \exp [-i(\omega+n \Omega) t]$, because their contribution is smaller than the kept ones. We see that the modulation of the longitudinal pumping yields the combined resonance, in each elementary process of which two energies of the elementary excitation of the quantum spin model are equal to the energy of the photon of the pumping plus the energy of $n$ photons of the modulating rf field.

Now we can use the unitary transformation $\rho_{1}=$ $U_{2} \rho_{2} U_{2}^{-1}$ with $U_{2}=\exp \left\{-i[(\omega+n \Omega) t / 2] \sum_{k} a_{k}^{\dagger} a_{k}\right\}$, which yields

$$
\begin{aligned}
i \hbar \dot{\rho}_{2}= & {\left[\mathcal{H}_{2}, \rho_{2}\right]+i \hbar \gamma U_{2}^{-1} U_{1}^{-1} \rho_{0} U_{1} U_{2} \exp (\gamma t), } \\
\mathcal{H}_{2}= & \sum_{k}\left(\left[\varepsilon_{k}-\frac{\hbar(\omega+n \Omega)}{2}\right] a_{k}^{\dagger} a_{k}\right. \\
& \left.+i \sum_{n} \frac{g \mu_{B} h B_{\mathbf{k}} J_{n}(z)}{\hbar \omega}\left[a_{-k} a_{k}-\text { H.c. }\right]\right) .
\end{aligned}
$$

The unitary transformation $U_{2}$ is used to remove the explicit time dependence from the Hamiltonian: It is equivalent to the transfer to the rotating frame and standard for the consideration of the time-dependent periodic permutations in spin systems, analogous to the Floquet case. There can be several values of $n=0, \pm 1, \pm 2, \ldots$ for which the value $\hbar(\omega+n \Omega) / 2$ is inside the band of $\varepsilon_{k}$. The choice of $n$ is, therefore, determined by that resonance condition in the framework of the resonance approximation used.

Finally, we can diagonalize the Hamiltonian $\mathcal{H}_{2}$ by using the Bogolyubov transformation, and then use the unitary transformation $\rho_{2}=U_{3} \rho_{0} U_{3}^{-1}$, where $U_{3}=\exp \left(-\frac{i}{\hbar} \mathcal{H}_{2} t\right)$. The solution for the time dependence of the density matrix can be presented in the form

$$
\begin{aligned}
& \rho=V(t) \rho_{0} V^{-1}(t) \exp (-\gamma t), \quad t \leqslant \gamma^{-1}, \\
& \rho=\gamma V(t) \int_{-\infty}^{0} d t^{\prime} V^{-1}\left(t^{\prime}\right) \rho_{0} V\left(t^{\prime}\right) e^{\gamma\left(t^{\prime}-t\right)} V^{-1}(t), \\
& t \gg \gamma^{-1},
\end{aligned}
$$

where $V(x)=U_{1}(x) U_{2}(x) U_{3}(x)$. The dynamics of the closed system for $\gamma=0$, or the open system in the dynamical regime $t \ll \gamma^{-1}$ is determined by the first line of Eq. (27), while the second line defines the steady-state regime (for the open system) at $t \gg \gamma^{-1}$.

\section{RESULTS FOR SPIN SYSTEMS WITH FERMIONIC EIGENSTATES}

Let us calculate the magnetization per site of the system caused by the modulated ac magnetic field. For the Kitaev model we limit ourselves to the ground state, which is fixed by the sector with all $\alpha_{j}=-1[34,36,38]$. It is related to the positive sign in front of $J_{z}$ in $A_{k}$. The homogeneous change of the sign of $\alpha_{j}$ due to the pumping can produce the negative sign of $J_{z}$ (homogeneously). In the following we consider the response of the system to the pumping for the fixed sign of $J_{z}$. Obviously, in the zero-temperature limit the density matrix $\rho_{0}$ is reduced to the ground-state wave function. To calculate the ac field-induced magnetization of the Kitaev system, we use the following relation [37]: For spin operators in the neighboring sites $j$ and $j^{\prime}$ of the lattice it is easy to show that $(1 / 2)\left(S_{j}^{z}+S_{j^{\prime}}^{z}\right)=2\left(\sqrt{2+2 \alpha_{j}}+2\left(\sqrt{2-2 \alpha_{j}}-\right.\right.$ $\left.\sqrt{2+2 \alpha_{j}}\right) d_{j}^{\dagger} d_{j}$. The proof of the relation uses the definition of spin operators and properties of the fermionic Majorana and Dirac operators.

Assume that the density matrix relaxes to the state $\rho_{0}$, which is the Gibbs distribution with $\mathcal{H}_{\text {1ind }}$ [19]. Let us define $\Delta M^{z} \equiv M^{z}-M_{0}^{z}$, where $M_{0}^{z}=(1 / 2 N)$ $\sum_{k} \tanh \left(\varepsilon_{k} / 2 k_{B} T\right)\left(A_{k} / \varepsilon_{k}\right)$ is the value of the magnetization per site for $h=h_{m}=0, N$ is the number of sites, 
$\Delta M^{z}=\sum_{n} M_{d}^{(n)} \exp (-\gamma t)$ in the dynamical regime, and $\Delta M^{z}=\sum_{n}^{n} M_{s}^{(n)}$ in the steady-state regime, where the sum is over all possible $n$, for which the resonance condition $\hbar(\omega+n \Omega)=2 \varepsilon_{k}$ holds.

We get

$$
\begin{aligned}
M_{d}^{(n)}= & \frac{g^{2} \mu_{B}^{2} h J_{n}(z) J_{0}^{2}(z)}{N \hbar \omega} \sum_{k} \tanh \left(\frac{\varepsilon_{k}}{2 k_{B} T}\right) \\
& \times \frac{\left|B_{k}\right|^{2}}{\varepsilon_{k} \hbar^{2} \Omega_{k}^{2}}\left\{[ 1 - \operatorname { c o s } ( 2 \Omega _ { k } t ) ] \left[-\frac{2 g \mu_{B} h A_{k} J_{n}(z)}{\hbar \omega}\right.\right. \\
& \left.+\left(\varepsilon_{k}-\frac{\hbar(\omega+n \Omega)}{2}\right) \cos [(\omega+n \Omega) t]\right] \\
& \left.+\hbar \Omega_{k} \sin \left(2 \Omega_{k} t\right) \sin [(\omega+n \Omega) t]\right\}
\end{aligned}
$$

We use the notation $\hbar \Omega_{k}=\left\{\left[\varepsilon_{k}-\hbar(\omega+n \Omega) / 2\right]^{2}+\right.$ $\left.\left(2 g \mu_{B} h / \hbar \omega\right)^{2}\left|B_{k}\right|^{2} J_{n}^{2}(z)\right\}^{1 / 2}$. In the steady-state regime we obtain

$$
\begin{aligned}
M_{s}^{(n)}= & \frac{4 g^{2} \mu_{B}^{2} h J_{n}(z) J_{0}^{2}(z)}{N \hbar \omega} \sum_{k} \tanh \left(\frac{\varepsilon_{k}}{2 k_{B} T}\right) \\
& \times \frac{\left|B_{k}\right|^{2}}{\varepsilon_{k} \hbar^{2}\left[\Omega_{k}^{2}+(\gamma / 2)^{2}\right]}\left\{\left[\frac{-2 g \mu_{B} h J_{0}(z) A_{k}}{\hbar \omega}\right.\right. \\
& \left.+\left(\varepsilon_{k}-\frac{\hbar(\omega+n \Omega)}{2}\right) \cos [(\omega+n \Omega) t]\right] \\
& \left.+\frac{\hbar \gamma}{2} \sin [(\omega+n \Omega) t]\right\}
\end{aligned}
$$

In both Eqs. (28) and (29) for the Kitaev honeycomb model we limit ourselves with the ground state, thus we replace

$$
\tanh \left(\frac{\varepsilon_{k}}{2 k_{B} T}\right) \rightarrow \frac{\varepsilon_{k}}{\left|\varepsilon_{k}\right|}
$$

In the thermodynamic limit $N \rightarrow \infty$ we replace $N^{-1} \sum_{k} \rightarrow$ $\pi^{-1} \int_{0}^{\pi} d k$ in the case of the spin chain, and $N^{-1} \sum_{k}^{k} \rightarrow$ $\left(2 \pi^{2}\right)^{-1} \int_{0}^{\pi} d k_{x} \int_{-\pi}^{\pi} d k_{y}$ for the two-dimensional Kitaev model. For the closed case we can use the expression for the dynamical regime, with $\gamma=0$.

We see that, in the dynamical regime $t \leqslant \gamma^{-1}$, the magnetization of the quantum spin system oscillates with the frequency $\omega+n \Omega$, modulated by the Rabi-like low frequencies $\Omega_{k}$. The latter in resonance are determined by the magnitude of the pumping, i.e., they are much smaller than $\omega+n \Omega$. Generically, closed interacting periodically driven quantum systems heat up to an infinite temperature state at long times (both for bosonic and fermionic systems). In our approach the density matrix of the system without external time-dependent perturbation was used as the initial condition (it is standard for similar problems). Namely, that procedure prevents the system from the heating to the infinite temperature state. On the other hand, in the steady-state regime $t \gg \gamma^{-1}$, relaxation "smears out" the Rabi-like oscillations, with only high frequency (with $\omega+n \Omega$ ) remaining. The magnitude of the oscillations for $n \neq 0$ is smaller than the main resonance (in the absence of modulation) for $n=0$.

The power of the ac magnetic field, absorbed by the quantum spin system is $Q=\langle\dot{\mathcal{H}}\rangle_{a v}$, where we use the definition of the time average $A_{a v}(t)=\lim _{\tau \rightarrow \infty} \tau^{-1} \int_{0}^{\tau} d t A(t)$. In the resonance approximation we obtain that, in the dynamical regime and for the closed system $Q \rightarrow 0$, and in the steadystate regime for the open system, we get $Q=\sum_{n} Q^{(n)}$ with

$$
\begin{aligned}
Q^{(n)}= & \frac{2 \gamma\left(g \mu_{B} h\right)^{2} J_{n}^{2}(z) J_{0}^{2}(z)(\omega+n \Omega)}{N \omega} \\
& \times \sum_{k} \tanh \left(\frac{\varepsilon_{k}}{2 k_{B} T}\right) \frac{\left|B_{k}\right|^{2}}{\varepsilon_{k} \hbar^{2}\left[\Omega_{k}^{2}+(\gamma / 2)^{2}\right]} .
\end{aligned}
$$

On the other hand, if we use the value $\rho_{0}$, determined by the Hamiltonian $\mathcal{H}_{0}$, then one has to replace the following in Eqs. (28)-(31):

$$
\tanh \left(\frac{\varepsilon_{k}}{2 k_{B} T}\right) \rightarrow \tanh \left(\frac{\varepsilon_{k}^{0}}{2 k_{B} T}\right)\left[1-\frac{2\left|B_{k}\right|^{2} A_{k}\left[J_{0}(z)-J_{0}^{2}(z)\right]}{\varepsilon_{k}^{2} \varepsilon_{k}^{0}} \sin ^{2}\left(\varepsilon_{k} t\right)\right]
$$

where $\varepsilon_{k}^{0}=\left(A_{k}^{2}+\left|B_{k}\right|^{2}\right)^{1 / 2}$. Obviously for $h_{m}=0$, i.e., for $z=0$ we obtain $\varepsilon_{k}=\varepsilon_{k}^{0}$, hence $\Delta M^{z}=M_{d}^{(0)}$ in the closed regime, and $\Delta M^{z}=M_{s}^{(0)}$ with $Q=Q^{(0)}$ in the steady-state open regime, as it must be.

\section{Several important particular cases}

We present here several results for two most important cases of quantum spin systems with fermionic eigenstates, namely, the one-dimensional Ising chain and the Kitaev honeycomb model. For the latter we limit ourselves with the ground state, with the fixed values of $\alpha_{j}$, see above. Below we consider the situation, in which $\rho_{0}$ is determined by the Hamiltonian $\mathcal{H}_{0}$.

For the Ising chain (i.e., $J_{y}=J_{z}=0$ ) we obtain for the case $2 g \mu_{B} H \gg J_{x}$ (i.e., the Ising chain is in the disordered state) in the steady-state regime (i.e., for the open case) for the steady-state value of the magnetic moment, caused by the pumping

$$
\Delta\left(M_{s}^{(n)}\right)_{a v} \approx-\frac{2 g \mu_{B} V^{2} J_{0}^{2}(z)}{\left(\Omega_{+}-\Omega_{-}\right)^{2}-4 V^{2}}\left[\frac{\left[\left(\Omega_{+}^{2}+\gamma^{2}\right)+\left(\Omega_{-}^{2}+\gamma^{2}\right)\right]^{1 / 2}}{\left[2 \sqrt{\left(\Omega_{+}^{2}+\gamma^{2}\right)\left(\Omega_{-}^{2}+\gamma^{2}\right)}+2 \Omega_{+} \Omega_{-}+2 \gamma^{2}+4 V^{2}\right]^{1 / 2}}-1\right],
$$


where $V=2 g \mu_{B} h J_{n}(z) J_{x} / \hbar \omega$ and $\hbar \Omega_{ \pm}=\hbar(\omega+n \Omega)-2 g \mu_{B} H \pm J_{x}$. The expression for the absorbed power of the pumping in this case can be written as

$$
Q^{(n)} \approx \frac{\gamma \hbar^{2}(\omega+n \Omega)^{2} V^{2} J_{0}^{2}(z)}{4 g \mu_{B} H\left[\left(\Omega_{+}-\Omega_{-}\right)^{2}-4 V^{2}\right]}\left[\frac{\left[\left(\Omega_{+}^{2}+\gamma^{2}\right)+\left(\Omega_{-}^{2}+\gamma^{2}\right)\right]^{1 / 2}}{\left[2 \sqrt{\left(\Omega_{+}^{2}+\gamma^{2}\right)\left(\Omega_{-}^{2}+\gamma^{2}\right)}+2 \Omega_{+} \Omega_{-}+2 \gamma^{2}+4 V^{2}\right]^{1 / 2}}-1\right] .
$$

For the opposite case $2 g \mu_{B} H \ll J_{x}$, i.e., the Ising chain is in the ordered state, the results for the steady-state magnetic moment, caused by the pumping, and the absorbed power of the pumping can be written in the form of Eqs. (33) and (34), respectively, with the replacements $\hbar \Omega_{ \pm} \rightarrow \hbar(\omega+$ $n \Omega)-J_{x} \pm 2 g \mu_{B} H$, and $4 g \mu_{B} H \rightarrow 2 J_{x}$ in the expression for the absorbed power. Notice that, at high temperatures $k_{B} T \gg$ $\max _{k}\left(\varepsilon_{k}^{0}\right)$, the expression for the absorbed power can be obtained for both limiting cases $2 g \mu_{B} H \gg J_{x}$ and $2 g \mu_{B} H \ll J_{x}$ by the replacements $4 g \mu_{B} H$ or $2 J_{x}$ by $8 k_{B} T$, respectively.

Figure 1 shows the behavior of the addition to the ground state magnetic moment of the Ising chain with $J_{x}=1$ at $H=$ 0 as a function of $g \mu_{B} h_{M}$ and time for the case of the closed system. For that situation, naturally only one value of $n$ is related to the resonance situation. We see that the oscillations in time with the frequency $\omega+n \Omega$ are modulated by the small Rabi frequency. Also, the magnitude of the time-dependent oscillations is modulated by the amplitude of the modulating field $h_{m}$.

On the other hand, Fig. 2 shows the behavior of the steadystate addition to the ground-state magnetic moment of the same Ising chain as a function of $g \mu_{B} h_{m}$ and time for the case of the open system. It is clearly seen that the modulation of oscillations with small Rabi-like frequency is smeared out. However the modulations, related to the interference of oscillations with frequencies $\omega$ and $2 \Omega$ are more pronounced, comparing with the closed system. Notice also smaller scale

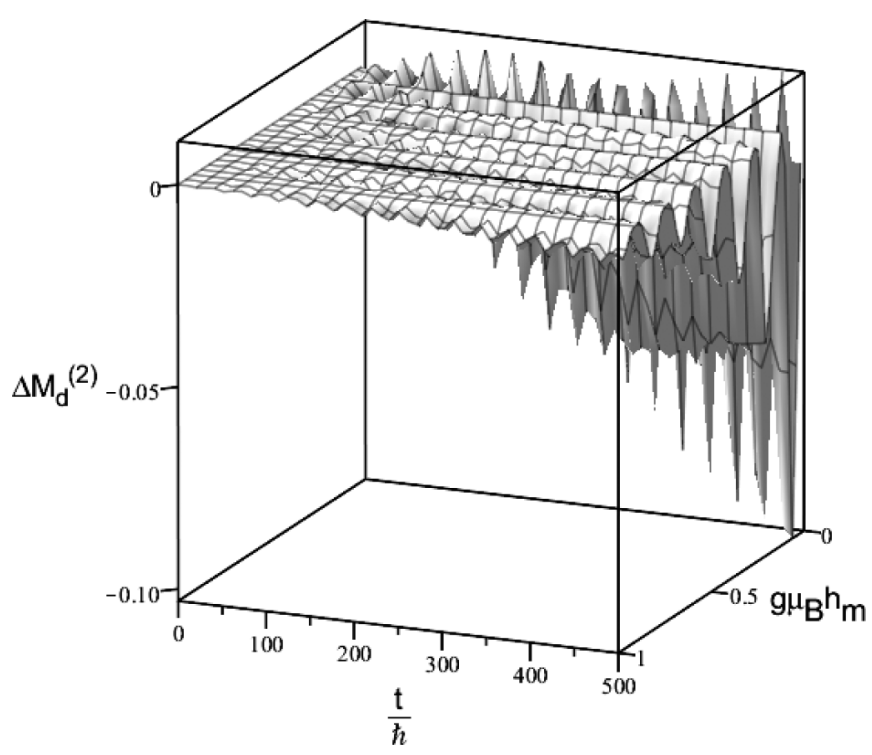

FIG. 1. The addition to the ground-state magnetic moment per site of the Ising chain in the case of the closed system with $J_{x}=1$, caused by the ac magnetic field. The used parameters are $g \mu_{B} h=$ $0.01, \hbar \omega=0.8, \hbar \Omega=0.1, n=2$. of oscillations of the magnetic moment, comparing with the open case. It turns out that, unlike the closed-system situation, the growth of the modulating amplitude $h_{m}$ does not cause such a strong decrease of the magnitude of the oscillations. Figures 3 and 4 present results of calculation of the absorbed power of the modulated ac magnetic field for the Kitaev honeycomb spin model at $T=0$ in the gapped regime $\left(J_{x}=\right.$ $\left.1, J_{y}=0.5, J_{z}=2\right)$ and in the gapless regime $\left(J_{x}=1, J_{y}=\right.$ $0.1, J_{z}=0.5$ ), respectively. For the Kitaev model we used the summation over all $\mathbf{k}$ belonging to the subset of the Brillouin zone such that $-\mathbf{k}$ is out of that subset [36]. We used the small value of the modulation frequency $\hbar \Omega=0.01$, so that the resonance conditions are satisfied for several values of $n$. In both figures one can clearly see oscillations of the absorbed power as a function of $h_{m}$. The values of additional peaks with respect to the case $h_{m}=0$, cf. [37] (corresponding to larger values of $n$ ) decay with $n$. Figure 3 manifests absorption for the frequency larger than the gap value, while in Fig. 4 the absorption exists for small values of $\omega$, including zero.

We see that, in the general situation the modulation of the pumping field results in the oscillating of the magnetic moment in time with the combined frequencies $\omega+n \Omega$ instead of $\omega$ for $h_{m}=0$. Also we see that the amplitude of the modulation of oscillations in time, as well as the renormalization of the steady-state value, about which oscillations in time persist, $\Delta M_{a v}^{z}$ oscillate with the value $2 g \mu_{B} h_{m} / \hbar \Omega$, decaying with $n$, as determined by the Bessel functions $J_{0}(z)$ and $J_{n}(z)$ [for all possible values of $n$, defined by the resonance condition $\left.\hbar(\omega+n \Omega)=2 \varepsilon_{k}\right]$. For $\Delta M^{z}$ both in the open and closed

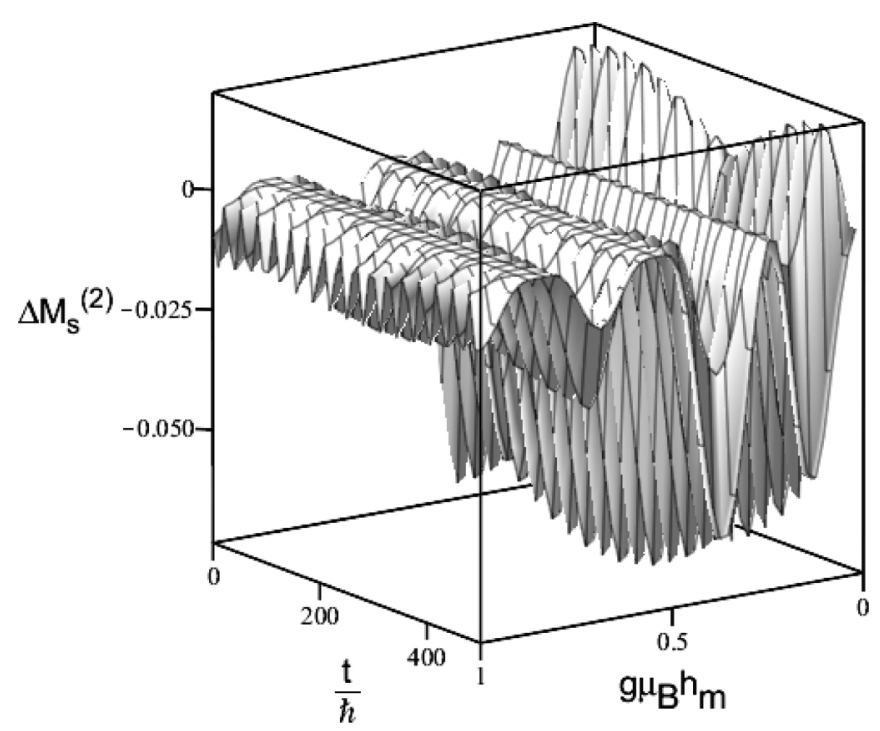

FIG. 2. The steady-state addition to the ground-state magnetic moment per site of the Ising chain for the case of the open system. The same parameters as in Fig. 1 are used with $\hbar \gamma=0.01$. 


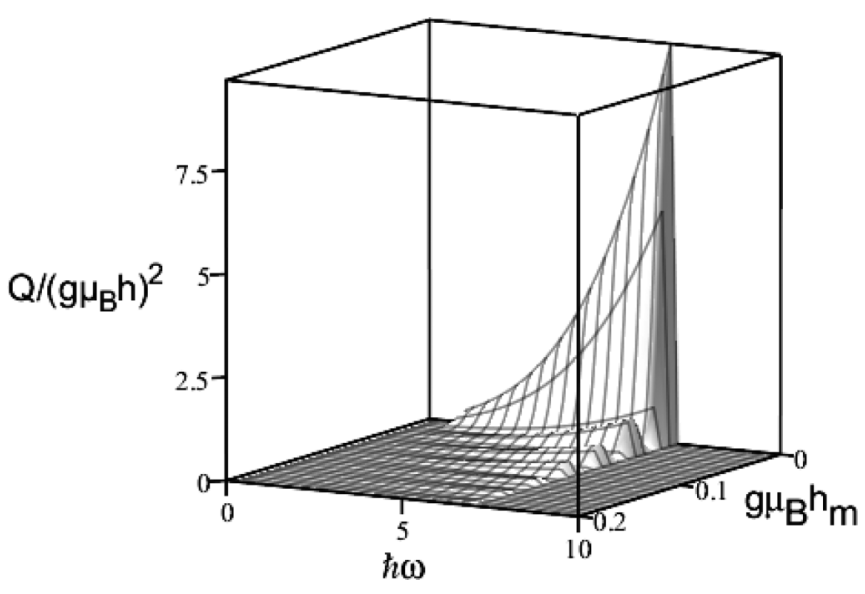

FIG. 3. Absorbed power of the modulated magnetic pumping field by the Kitaev honeycomb spin model at $T=0$ in the gapped regime $\left(J_{x}=1, J_{y}=0.5, J_{z}=2\right)$. The frequency of the modulated field is $\hbar \Omega=0.01$.

regimes we have for the case of the density matrix determined by the Hamiltonian $\mathcal{H}_{1 \text { ind }}$,

$$
\frac{\Delta M_{a v}^{z}}{\Delta M_{a v}^{z}\left(h_{m}=0\right)} \approx J_{0}^{2}(z) \sum_{n} J_{n}(z)
$$

and for the absorbed power in the open regime,

$$
\frac{Q}{Q\left(h_{m}=0\right)} \approx J_{0}^{2}(z) \sum_{n} J_{n}^{2}(z)
$$

where the summations are over all possible values of $n$, determined by the resonance condition. Notice that $J_{-n}(z)=$ $-J_{n}(z)$, so the contributions of the combined resonances with equal positive and negative values of $n$ are less pronounced for the magnetization, but not for the absorbed power, proportional to the square of the Bessel function. In addition, in resonance the magnitude of oscillations with the frequencies $\omega+n \Omega$ oscillates with $g \mu_{B} h_{m} / \hbar \Omega$ in

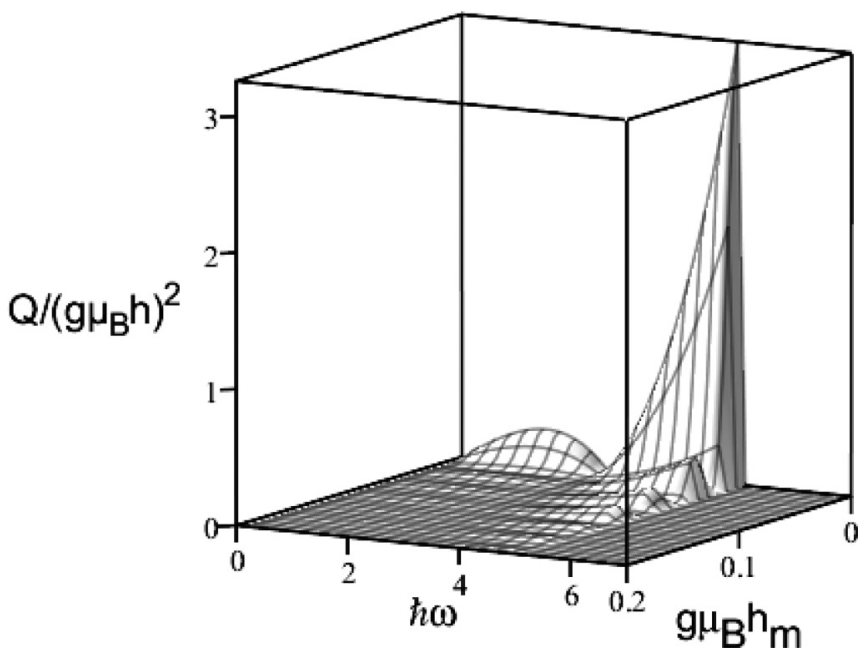

FIG. 4. The same as in Fig. 3 but for the Kitaev model in the gapless regime $\left(J_{x}=1, J_{y}=0.1, J_{z}=0.5\right)$.

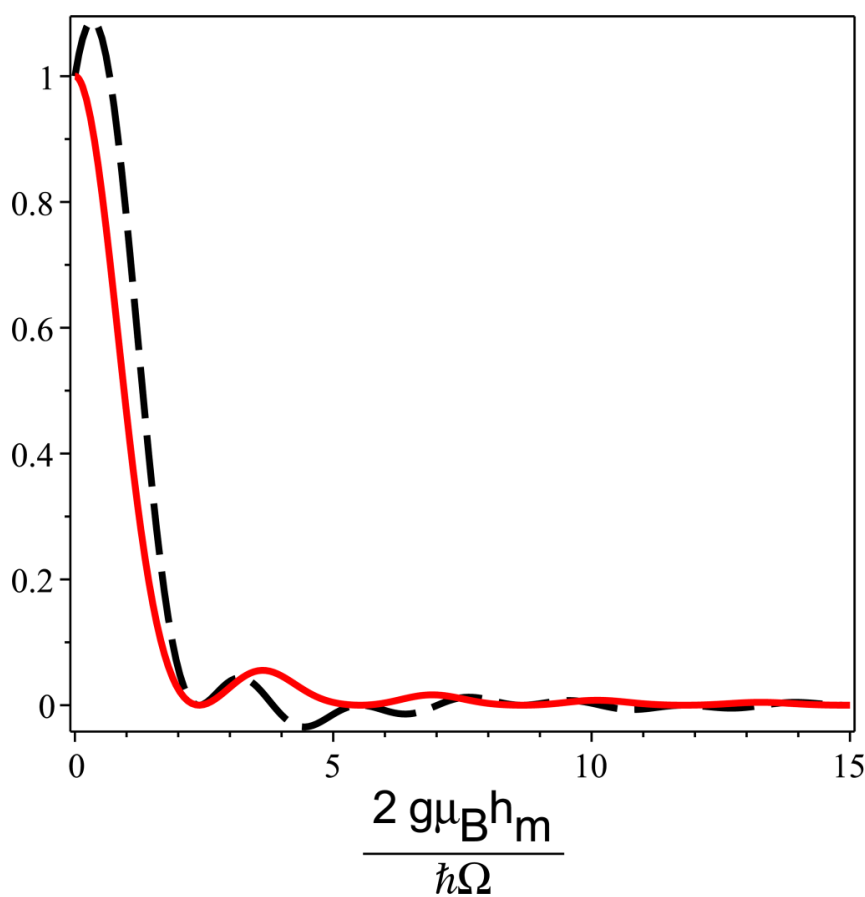

FIG. 5. The relative values of the averaged-in-time addition to the magnetic moment caused by the parallel pumping $\Delta M_{a v}^{z} / \Delta M_{a v}^{z}\left(h_{m}=0\right)$ (the dashed black curve) and the absorbed power $Q / Q\left(h_{m}=0\right)$ (the solid red curve) of the quantum spin system with fermionic eigenstates as a function of $2 g \mu_{B} h_{m} / \hbar \Omega$ for $n=0,1,2$.

both closed and open regimes. Also, in the closed regime the frequencies of the Rabi-like oscillations in resonance are $\left(2 g \mu_{B} h / \hbar \omega\right)\left|B_{k}\right|\left|J_{n}(z)\right| \ll \omega+n \Omega$, and the magnitudes of those time-dependent oscillations also oscillate with $g \mu_{B} h_{m} / \hbar \Omega$. For example, if only $n=0,1,2$ satisfy the resonance condition, the behavior of $\Delta M_{a v}^{z} / \Delta M_{a v}^{z}\left(h_{m}=0\right)$ and $Q / Q\left(h_{m}=0\right)$ is shown in Fig. 5.

Notice that if $\Delta M_{a v}^{z}$ or $Q$ are equal to zero for some values of $g \mu_{B} h_{m} / \hbar \Omega$, those values are determined by the small nonresonance terms $\approx g \mu_{B} h / \hbar \omega$, which can be taken into account within the perturbation theory.

We stress that the modulation of the ac pumping field for quantum spin systems with fermionic eigenstates (which carry fractional spin $1 / 2$, unlike magnons or spin waves, which carry spin 1) yields modulation of the magnitudes and frequencies (in the closed regime) of infinitely many oscillations in time of all possible modes, interference of which produces a complicated picture, with possible decay of oscillations even for the closed system and in the dynamical regime for the open system, see below. Such a behavior is, probably, characteristic for quantum spin liquids with fermionic eigenstates. On the other hand, for magnetically ordered spin systems (or for disordered ones) with bosonic eigenstates, the modulation of the pumping field results in the oscillation of the threshold of the parametric instability of spin waves or magnons, above which the number of magnons or spin waves grows exponentially with time (for the noninteracting magnons such a growth cannot be limited by the linear relaxation). 


\section{SUMMARY}

In summary, we have shown that the modulation of a high-frequency ac magnetic field parallel to the dc one by the lower-frequency ac field, acting on quantum systems, produces combined resonances with the resonance condition $\hbar(\omega+n \Omega)=\varepsilon_{k}$, where $\varepsilon_{k}$ is the energy of fermion eigenstates, and the modulation of their characteristics as a function of the ratio of the magnitude and the frequency of the modulating field. When obtaining the results, the condition $g \mu_{B} h \ll \hbar \omega$ was used. For most of magnetically ordered spin systems, the modulation of the longitudinal pumping yields the oscillation of the threshold of the parametric instability of spin waves as a function of the amplitude and the frequency of the modulation. For quantum spin systems with fermionic eigenstates the modulating field causes only modulation of the time-dependent oscillations of the magnetization and the absorbtion of the system power of the ac field, unlike the exponential growth of the number of spin waves for magnetically ordered systems. Two main examples of such quantum spin systems are spin- $1 / 2$ chains (notice that interaction between fermions in our study was taken into account at the meanfield level), and honeycomb Kitaev spin model (in the ground state). We have shown that the magnetic moment for the closed system oscillates in time with the combined frequency of the pumping and $n$ frequencies of the modulation, and the smaller Rabi-like frequency. Similar behavior is shown to persist for the dynamical regime of the open system. On the other hand, in the steady-state regime of the open system, the Rabi-like oscillations are damped. We predict that the magnitudes of all mentioned time-dependent oscillations have to be modulated by the modulation if field. We have also shown that the power of the ac field, absorbed by the quantum spin system, also oscillates as a function of the magnitude and the frequency of the modulating field. The oscillation of the magnetic moment and absorbed power for the combined resonances can help to clarify the characteristics of quantum spin systems. Fermionic eigenstates, which carry fractional spin, are characteristic for many quantum spin liquids, and we suppose that the effect of modulation of the longitudinal pumping in quantum spin liquids with fermionic excitations will be similar to the one considered in our study.

\section{ACKNOWLEDGMENTS}

I thank N. Konik for the helpful suggestions. The support from the Deutsche Forschungsgemeinschaft via the SFB 1143 is acknowledged.
[1] J. M. Deutsch, Phys. Rev. A 43, 2046 (1991); M. Srednicki, Phys. Rev. E 50, 888 (1994); M. Rigol and M. Srednicki, Phys. Rev. Lett. 108, 110601 (2012); M. Rigol, V. Dunjko, and M. Olshanii, Nature (London) 452, 854 (2008).

[2] M. Moeckel and S. Kehrein, Phys. Rev. Lett. 100, 175702 (2008); Ann. Phys. 324, 2146 (2009).

[3] M. Rigol, V. Dunjko, V. Yurovsky, and M. Olshanii, Phys. Rev. Lett. 98, 050405 (2007).

[4] For a review, see A. A. Zvyagin, Fiz. Nizk. Temp. 42, 1240 (2016) [Low Temp. Phys. 42, 971 (2016)]; M. Heyl, Rep. Prog. Phys. 81, 054001 (2018).

[5] G. Floquet, Ann. Sci. Ecole Norm. Sup. 12, 47 (1883).

[6] I. Bloch, J. Dalibard, and W. Zwerger, Rev. Mod. Phys. 80, 885 (2008); P. Ponte, Z. Papic, F. Huveneers, and D. A. Abanin, Phys. Rev. Lett. 114, 140401 (2015); A. Lazarides, A. Das, and R. Moessner, ibid. 115, 030402 (2015); P. Bordia, H. Lüschen, U. Schneider, M. Knap, and I. Bloch, Nat. Phys. 13, 460 (2017).

[7] See, e.g., B. Ferguson and X.-C. Zhang, Nat. Mater 1, 26 (2002); M. Tonouchi, Nat. Photon. 1, 97 (2007); T. Kampfrath, A. Sell, G. Klatt, A Pashkin, S. Mährlein, T. Dekorsy, M. Wolf, M. Fiebig, A. Leitenstorfer, and R. Huber, ibid. 5, 31 (2011); B. E. Cole, J. B. Williams, B. T. King, M. S. Sherwin, and C. R. Stanley, Nature (London) 410, 60 (2001); R. Huber, F. Tauser, A. Brodschelm, M. Bichler, G. Abstreiter, and A. Leitenstorfer, ibid. 414, 286 (2001); R. A. Kaindl, M. A. Carnahan, D. Hägele, R. Lövenich, and D. S. Chemla, ibid. 423, 734 (2003); S. G. Carter V. Birkedal, C. S. Wang, L. A. Coldren, A. V. Maslov, D. S. Citrin, and M. S. Sherwin, Science 310, 651 (2005); J. Kröll, J. Darmo, S. S. Dhillon, X. Marcadet, M. Calligaro, C. Sirtori, and K. Unterrainer, Nature (London) 449, 698 (2007); J. R. Danielson, Y.-S. Lee, J. P. Prineas, J. T. Steiner, M. Kira, and S. W. Koch, Phys. Rev. Lett. 99, 237401
(2007); T. Kinoshita, T. Wenger, and D. S. Weiss, Nature (London) 440, 900 (2006); M. Gring, M. Kuhnert, T. Langen, T. Kitagawa, B. Rauer, M. Schreitl, I. Mazets, D. A. Smith, E. Demler, and J. Schmiedmayer, Science 337, 1318 (2012).

[8] See, e.g., S. Zherlitsyn, B. Wustmann, T. Herrmannsdörfer, and J. Wosnitza, IEEE Trans. Appl. Supercond. 22, 4300603 (2012).

[9] For a review see, e.g., H. Häffner, C. F. Roos, and R. Blatt, Phys Rep. 469, 155 (2008).

[10] See, e.g., A. A. Zvyagin, Quantum Theory of One-Dimensional Spin Systems (Cambridge Scientific Publishers, Cambridge, 2010).

[11] N. D. Mermin and H. Wagner, Phys. Rev. Lett. 17, 1133 (1966).

[12] S. Kimura, T. Takeuchi, K. Okunishi, M. Hagiwara, Z. He, K. Kindo, T. Taniyama, and M. Itoh, Phys. Rev. Lett. 100, 057202 (2008); S. Agrestini, C. L. Fleck, L. C. Chapon, C. Mazzoli, A. Bombardi, M. R. Lees, and O. A. Petrenko, ibid. 106, 197204 (2011).

[13] For a review, see, e.g., J. Alicea, Rep. Prog. Phys. 75, 076501 (2012); C. W. J. Beenakker, Annu. Rev. Condens. Matter Phys. 4, 113 (2011); T. D. Stanescu and S. Tewari, J. Phys.: Condens. Matter 25, 233201 (2013).

[14] See, e.g., U. Divakaran and A. Dutta, Phys. Rev. B 79, 224408 (2009); A. A. Zvyagin, ibid. 90, 014507 (2014); R. Vasseur, J. P. Dahlhaus, and J. E. Moore, Phys. Rev. X 4, 041007 (2014); A. Rajak, T. Nag, and A. Dutta, Phys. Rev. E 90, 042107 (2014); P. D. Sacramento, ibid. 90, 032138 (2014); G. Kells, D. Sen, J. K. Slingerland, and S. Vishveshwara, Phys. Rev. B 89, 235130 (2014).

[15] A. Friedenauer, H. Schmitz, J. T. Glueckert, D. Porras, and T. Schaetz, Nat. Phys. 4, 757 (2008); K. Kim, M.-S. Chang, R. Islam, S. Korenblit, L.-M. Duan, and C. Monroe, Phys. Rev. Lett. 103, 120502 (2009); K. Kim, M.-S. Chang, S. Korenblit, 
R. Islam, E. E. Edwards, J. K. Freericks, G.-D. Lin, L.-M. Duan, and C. Monroe, Nature (London) 465, 590 (2010); B. P. Lanyon, C. Hempel, D. Nigg, M. Müller, R. Gerritsma, F. Zähringer, P. Schindler, J. T. Barreiro, M. Rambach, G. Kirchmair, M. Hennrich, P. Zoller, R. Blatt, and C. F. Roos, Science 334, 57 (2011); R. Islam, E. Edwards, K. Kim, S. Korenblit, C. Noh, H. Carmichael, G.-D. Lin, L.-M. Duan, C.-C. J. Wang, J. Freericks, and C. Monroe, Nat. Commun. 2, 377 (2011); J. W. Britton, B. C. Sawyer, A. C. Keith, C.-C. J. Wang, J. K. Freericks, H. Uys, M. J. Biercuk, and J. J. Bollinger, Nature (London) 484, 489 (2012); P. Richerme, C. Senko, S. Korenblit, J. Smith, A. Lee, R. Islam, W. C. Campbell, and C. Monroe, Phys. Rev. Lett. 111, 100506 (2013); R. Islam, C. Senko, W. C. Campbell, S. Korenblit, J. Smith, A. Lee, E. E. Edwards, C.-C. J. Wang, J. K. Freericks, and C. Monroe, Science 340, 583 (2013).

[16] P. A. Lee, Science 321, 1306 (2008); L. Savary and L. Balents, Rep. Prog. Phys. 80, 016502 (2017).

[17] G. Lindblad, Commun. Math. Phys. 48, 119 (1976); V. Gorini, A. Kossakovski, and E. C. G. Sudarshan, J. Math. Phys. 17, 821 (1976).

[18] Y. A. Ilyinskiy and L. V. Keldysh, Interaction of Electromagnetic Radiation with Matter (in Russian) (Moscow State University Publishers, Moscow, 1989).

[19] R. Karplus and J. Schwinger, Phys. Rev. 73, 1020 (1948).

[20] F. Bloch, Phys. Rev. 70, 460 (1946).

[21] H. C. Torrey, Phys. Rev. 104, 563 (1956).

[22] A. A. Zvyagin, Fiz. Nizk. Temp. 41, 938 (2015) [Low Temp. Phys. 41, 730 (2015)].

[23] T. Holstein and H. Primakoff, Phys. Rev. 58, 1098 (1940).

[24] F. R. Morgenthaller, J. Appl. Phys. 31, S95 (1960); E. Schlömann, J. J. Green, and U. Milano, ibid. 31, S386 (1960).

[25] V. E. Zakharov, V. S. L'vov, and S. S. Starobinets, Sov. Phys. Usp. 17, 896 (1975).
[26] A. A. Zvyagin, Fiz. Nizk. Temp. 42, 370 (2016) [Low Temp. Phys. 42, 286 (2016)].

[27] See, e.g., L. W. Hinderks and P. M. Richards, J. Appl. Phys. 39, 824 (1968); M. H. Seavey, ibid. 40, 1597 (1969); Phys. Rev. Lett. 23, 132 (1969); W. Jantz, J. Schneider, and B. Andlauer, Solid State Commun. 10, 937 (1972); S. O. Demokritov, V. E. Demidov, O. Dzyapko. G. A. Melkov. A. A. Serga, B. Hillebrands, and A. N. Slavin, Nature (London) 443, 430 (2006); T. Neumann, A. A. Serga, V. I. Vasyuchka, and B. Hillebrands, Appl. Phys. Lett. 94, 195202 (2009).

[28] H. Suhl, Phys. Rev. Lett. 6, 174 (1961); A. M. Frishman, Fiz. Nizk. Temp. 8, 554 (1982).

[29] T. S. Hartwick, E. R. Peressini, and M. T. Weiss, Phys. Rev. Lett. 6, 176 (1961); V. I. Ozhogin, S. M. Suleimanov, and A. Yu. Yakubovskii, Pis'ma v Zh. Eks. Teor. Fiz. 32, 308 (1981) [JETP Lett. 32, 284 (1981)].

[30] P. Jordan and E. Wigner, Eur. Phys. J. A 47, 631 (1928).

[31] A. A. Zvyagin, Phys. Rev. B 92, 184507 (2015).

[32] See, e.g, P. Pfeuty, Ann. Phys. (NY) 57, 79 (1970); E. Barough and B. McCoy, Phys. Rev. A 3, 786 (1973); E. Barough, B. McCoy, and T. T. Wu, Phys. Rev. Lett. 31, 1409 (1973); S. Sachdev, Quantum Phase Transitions (Cambridge University Press, Cambridge, 2011).

[33] A. Luther and I. Peschel, Phys. Rev. B 12, 3908 (1975).

[34] A. Kitaev, Ann. Phys. (NY) 321, 2 (2006).

[35] X.-Y. Feng, G.-M. Zhang, and T. Xiang, Phys. Rev. Lett. 98, 087204 (2007); H.-D. Chen and J. Hu, Phys. Rev. B 76, 193101 (2007); H.-D. Chen and Z. Nussinov, J. Phys. A: Math. Theor. 41, 075001 (2008).

[36] M. Schmitt and S. Kehrein, Phys. Rev. B 92, 075114 (2015).

[37] A. A. Zvyagin, Phys. Rev. B 95, 064428 (2017).

[38] E. H. Lieb, Phys. Rev. Lett. 73, 2158 (1994). 